\title{
Mean platelet volume: A simple tool for early risk stra- tification of pulmonary embolism
}

\author{
Ayşegül Şentürk ${ }^{1^{*}}$, Asiye Kanbay ${ }^{2}$, Serpil Erdoğan ${ }^{3}$, Hatice Kılıç ${ }^{1}$, Tuba Öğüt ${ }^{4}$, \\ Habibe Hezer ${ }^{1}$, Hatice Canan Hasanoğlu ${ }^{5}$ \\ 1 Ataturk. Training and Research Hospital, Department of Pulmonary Diseases, Ankara, Turkey \\ ${ }^{2}$ Istanbul Medeniyet University School of Medicine, Department of Pulmonary Medicine, Istanbul, Turkey \\ ${ }^{3}$ Ataturk Training and Research Hospital, Department of Clinical Biochemistry, Ankara, Turkey \\ ${ }^{4}$ Igdir State Hospital, Clinics of Pulmonary Diseases, Igdir, Turkey \\ ${ }^{5}$ Yildirim Beyazid University School of Medicine, Department of Pulmonary Medicine, Ankara, Turkey
}

\begin{abstract}
Pulmonary embolism (PE) is still a major health problem associated with a significant mortality and morbidity. Rapid recognition of massive and submassive cases should be easy and available in order to commencement of appropriate therapy. The aim of this study is to assess the relationship between mean platelet volume (MPV) and PE severity.

Three hundred twenty five patients with proven PE (massive $=113$, submassive $=129$, and non-massive $=83$ patients) groups were retrospectively evaluated. The mean age of the participants was 68 (range: 21-93) years. The distribution of age was different among PE and control cases.

The MPV value of patients with PE group was significantly higher compared to healthy subjects $9.8 \pm 1.7 \mathrm{fl} \mathrm{vs} .9 .1 \pm 0.8 \mathrm{fl}$, $\mathrm{p}<0.001)$. There were significant differences in regards to MPV levels between the patients with massive, sub-massive, and non-massive PE $[10(6.9-17.2)$ vs. 9.8 (6.6-19.5) vs. 9.2(5.8-13.2) fl, respectively $p=0.004]$.

MPV is significantly higher in subjects with newly diagnosed PE patients and also MPV is strongly associated with the severity of PE.
\end{abstract}

Key Words: Pulmonary embolism severity, mean platelet volume, platelet functions

\section{Introduction}

Despite advances in diagnosis and treatment of pulmonary embolism (PE), it is still one of the most important causes of mortality and morbidity in all over the world. Determination of risk stage of the PE is not always easy since its clinical signs rapidly change from asymptomatic disease to death (1). In patients with the suspicion of PE, first of all risk stratification should be performed. Especially among hemodynamically stable patients, in determination of high risk group, echocardiographic findings and cardiac markers are commonly used $(2,3,4)$. However, it is not always easy to attain these procedures. In patients with PE, inexpensive, available and practical tools are necessary to determine prognosis and treatment strategies. Due to this, some investigators focused on about the roles of thrombocytes in thrombus formation (5).

Thrombocytes play a central role in pathogenesis of vascular diseases. The width of thrombocytes are described as mean thrombocyte volume (MPV) and measured with automatic cellular counters routinely. Mean thrombocyte volume is a physiological variable with hemostatic significance. Large thrombocytes are known as more reactive, they produce more prothrombotic factors and they form clusters more easily than smaller ones. Moreover large thrombocytes contain dense granules and secrete serotonin and $\beta$-trombo-globulin in larger amounts compared with smaller ones. An association between increased platelet volumes and acute myocardial infarction, acute cerebral ischemia and transient ischemic attack has been reported $(5,6)$. Furthermore, an increase in mean platelet volume has been determined as an independent risk factor for recurrent vascular events and death after myocardial infarction. Also, increase in thrombocyte volumes has been reported in patients with renal artery stenosis and patients with vascular risk factors such as diabetes, hypercholesterolemia, and smoking $(7,8)$. Kostrubiec et al. (9) reported that MPV is an independent predictor of early death in acute PE. They concluded that high volumes of trombocytes are associated 
with right ventricular dysfunction and myocardial injury in acute PE. However none of the previous study is investigated the predictive value of MPV in diagnosing the severity of PE.

With this background in mind, we aimed to determine the effects of increased thrombocyte functions in development of thrombosis with severity of $\mathrm{PE}$ and to investigate the association of thrombocyte volume with PE.

\section{Materials and methods}

Three-hundred-twenty-five patients who were hospitalized with the diagnosis of PE in Pulmonary Disease Department between January 2009 December 2013 and 155 control cases were retrospectively evaluated. Control cases were selected among the patients without any risk factors for vascular disease and who admitted to our hospital for routine control in the same time period. Patients with the history of severe heart failure, myocardial infarction, atherosclerotic heart disease, peripheral artery disease, and chronic obstructive pulmonary disease (COPD), patients with the signs of any infection and patients who had hematological disease which affected platelets were excluded from the study. Patients using any drug (including aspirin, dipiradamol, clopidogrel, heparin, aminophylline, verapamil, nonsteroidantiinflammatory drugs, steroid, furosemide, antibiotics, and alcohol intake) that may affect thrombocyte functions were also excluded. Diagnosis of PE was established with computed tomography angiography. Severity of PE was classified in three groups according to The European Society of Cardiology (ESC) guidelines: High-risk (patients with shock or hypotension), intermediate-risk [(presence of positive marker for myocardial injury or right ventricle dysfunction (RVD)], lowrisk (absence of RVD or negative marker of myocardial injury) (10). Results of peripheral complete blood count (hemoglobin, thrombocyte count, white blood cell count, MPV), troponin and proBNP values of the venous blood samples, obtained from all cases in the first 24 hours of hospital admission and stored in EDTA tubes, were recorded. The blood samples were studied with the Sysmex-XE 2100 automated blood cell analyzer (Sysmex, Sweden). The expected normal values for MPV in our hematology laboratory ranged from 7.2 to $11.1 \mathrm{fl}$.

Statistical analysis: The software package SPSS for Windows version 17.0 (SPSS Inc., Chicago, III., USA) was used for statistical analyses. The Kolmogorov-Smirnov test was applied to determine the probability distribution. Data were reported as proportions or as mean \pm SD. Kruskal Wallis test was used for comparing the groups. The chi-square test was used for comparisons of discrete variables. A $P$-value $<0.05$ indicates statistical significance in all analyses. The following variables were considered in the multivariable analysis: demographic features (age, sex), comorbidity (cancer), laboratory findings (serum troponin level), right ventricle dysfunction (RVD) on echocardiography, and computerized tomography (CT) scan findings.

\section{Results}

The demographic features and clinical characteristics of study participants are summarized in Table 1 .

The mean age of the participants was 68 (range: 2193) years. The distribution of age was different

Table 1. Demographics and general characteristics of participants

\begin{tabular}{lccc}
\hline & $\begin{array}{c}\text { PE group } \\
(\mathrm{n}=325)\end{array}$ & $\begin{array}{c}\text { Control group } \\
(\mathrm{n}=155)\end{array}$ & P value \\
\hline Age (years) & $69(21-93)^{*}$ & $65(32-90)^{*}$ & 0.038 \\
\hline Sex, female $(\%)$ & $173(65 \%)$ & $93(35 \%)$ & 0.170 \\
\hline Smoking $[\mathrm{n}(\%)]$ & $91(28 \%)$ & 0 & \\
\hline Diabetes $[\mathrm{n}(\%)]$ & $42(12.9 \%)$ & 0 & $<0.001$ \\
\hline MPV $(\mathrm{fl})$ & $9.8 \pm 1.7$ & $9.1 \pm 0.8$ & $<0.001$ \\
\hline Platelet & $226(68-528)^{*}$ & $254(116-504)^{*}$ & $<0.001$ \\
\hline Total-cholesterol $(\mathrm{mg} / \mathrm{dl})$ & $170(14-738)^{*}$ & $198(108-324)^{*}$ & $<0.001$ \\
\hline HDL-cholesterol $(\mathrm{mg} / \mathrm{dl})$ & $36(10-72)^{*}$ & $49(25-100)^{*}$ & $<0.001$ \\
\hline LDL-cholesterol $(\mathrm{mg} / \mathrm{dl})$ & $101.8 \pm 36$ & $122 \pm 50$ &
\end{tabular}

[Median (min.-max)] *, MPV; mean platelet volume, PE; pulmonary embolism 
among PE and control cases. Median ages (min-max) of acute PE and control groups were 69 (21-93) years and 65 (32-90) years, respectively $(\mathrm{p}=0.038)$.

There were 155 (32.5\%), 83 (17.3\%), 129 (26.7\%), and $113(23.5 \%)$ participants in the control, nonmassive, sub-massive, and massive embolism groups, respectively. All patients were treated with heparin and $121(25.2 \%)$ patients with massive and sub-massive PE received additional thrombolytic therapies. All patients also received warfarin throughout the follow-up.

The mean MPV level of patients with acute PE group was significantly higher compared to healthy control subjects $9.8 \pm 1.7 \mathrm{fl}$ vs. $9.1 \pm 0.8 \mathrm{fl}$, $\mathrm{p}<0.001)$. All blood cell distribution parameters of both groups are summarized in Figure 1.

There were significant differences in terms of. MPV levels between the patients with massive, sub-massive, and non-massive acute PE [10 (6.9-
17.2) vs. $9.8(6.6-19.5)$ vs. 9.2(5.8-13.2)fl, respectively] (Table 2).

When we compared MPV values with echocardiographic findings, there was no correlation between MPV levels and right ventricular (RV) dysfunction, sPAB or pro-BNP values. Platelet count was negatively correlated with MPV $(r=-0.231$, $p=<.0001)$. There was an inverse correlation between systolic blood pressure and MPV $(r=.32$, $\mathrm{p}=0.577$ ). Moreover, there was a significant correlation between the mean MPV levels and Troponin-I $(r=0.549, p=<0.0001)$ (Table 3).

\section{Discussion}

The salient finding of the present is higher in subjects with newly diagnosed PE patients and also MPV is strongly associated with the severity of PE.

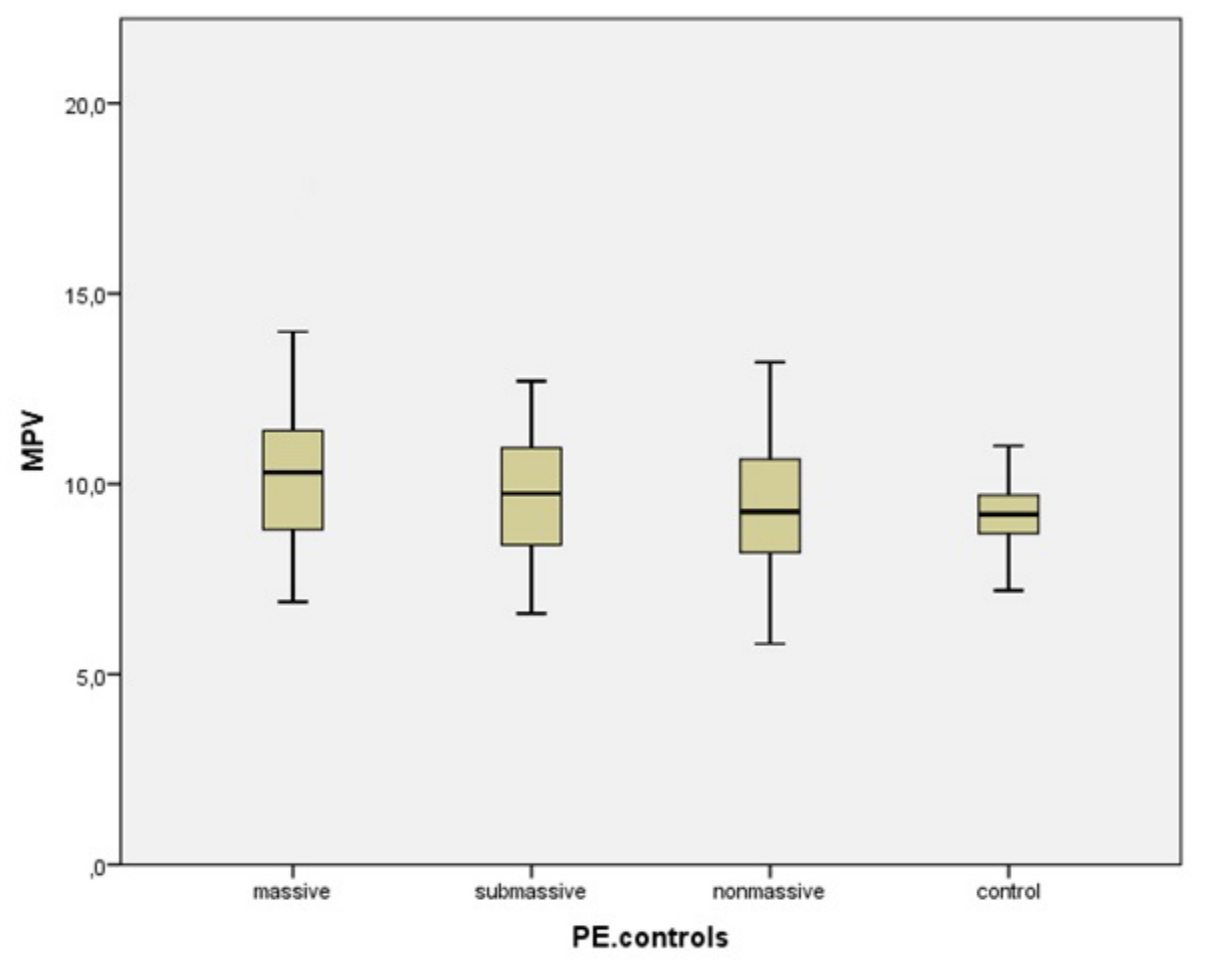

Fig. 1. MPV levels of study groups in massive, submassive, non-massive pulmonary embolism and control subjects.

Table 2. Clinical and laboratory characteristics of pulmonary embolism patients according to severity of pulmonary embolism

\begin{tabular}{lcccc}
\hline & $\begin{array}{c}\text { Massive PE } \\
(\mathrm{n}=113)\end{array}$ & $\begin{array}{c}\text { Submassive PE } \\
(\mathrm{n}=129)\end{array}$ & $\begin{array}{c}\text { Non-massive PE } \\
(\mathrm{n}=83)\end{array}$ & $\mathrm{P}$ \\
\hline Age (years) & $70(30-799)$ & $70(21-90)$ & $59(22-93)$ & $<0.0001$ \\
Troponin $(\mathrm{ng} / \mathrm{ml})^{*}$ & $0.12(0.01-0.7)$ & $0.07(0-9.7)$ & $0.01(0-0.1)$ & 0.201 \\
NT-proBNP & $1730(80320-0)$ & $880(15983-4.5)$ & $100(4760-0)$ & $<0.0001$ \\
MPV & $10(6.9-17.2)$ & $9.8(6.6-19.5)$ & $9.2(5.8-13.2)$ & 0.004 \\
\hline
\end{tabular}

East J Med Volume:22, Number:2, April-June/2017 
Tablo 3. Correlations of laboratory findings with the severity of pulmonary embolism

\begin{tabular}{lcc}
\hline Parameters & $\mathrm{r}$ & $p$ value \\
\hline Troponin $(\mathrm{ng} / \mathrm{ml})$ & -0.079 & 0.231 \\
NT-proBNP & -0.271 & $<0.001$ \\
MPV & -0.183 & $<0.001$ \\
Platelet & 0.242 & $<0.001$ \\
Sa O2, \% & 0.415 & $<0.001$ \\
WBC & -0.032 & 0.570 \\
sPAP & -0.415 & $<0.001$ \\
SBP & 0.248 & $<0.001$ \\
RVD & -0.488 & $<0.001$ \\
\hline
\end{tabular}

MPV; mean platelet volume, WBC; white blood cell, sPAP; systolic pulmonary artery pressure $(\mathrm{mmHg})$, SBP; systolic blood pressure, RVD; right ventricular dysfunction, $\mathrm{Sa} \mathrm{O}_{2}$; oxygen saturation in arterial blood.

Thrombocytes play a major role in the pathogenesis of athero-trombus formation. The alterations in the volume parameters of thrombocytes may be important in both prophylaxis and diagnosis of thrombotic and pre-thrombotic events (5). In this study we have determined a significant increase in MPV levels among PE patients compared with the control cases. We showed that MPV may be used as a marker for prediction of severity of acute PE.

Thrombocyte volume is a parameter determining thrombocyte function. Larger thrombocytes are more active in terms of hemostatic functions. Alterations in thrombocyte diameters and activities may influence the thrombus formation and development of acute myocardial infarct (11). In patients with acute myocardial infarct that have increased mean platelet volumes at admission, mortality and revascularization requirement was reported to be higher and it has been also been stated that follow up and treatment of these cases require more care $(12,13)$.

Platelet activation has been observed after acute PE. Whether platelet activation is a cause or consequence of acute PE in humans is unknown. However, platelet activation correlates with the severity of RV dysfunction, and can persist for several months after acute PE (14).

In an other study, mean MPV levels were determined to be similar in PE and control cases. However, MPV has been reported to be correlated with parameters of RV dysfunction and myocardial damage reflecting the severity of PE (9). On the other hand, in similar with this study, Ermis et al. (15) reported that MPV was not an indicator in diagnosis or determination of severity of acute PE however, they also reported that MPV was higher among acute PE. In contrast in our study, similar to the results of Varol et al. (16), the MPV was reported to be increased among acute PE patients, as compared to controls. Moreover, MPV was higher in massive PE patients compared to the patients with sub-massive or non-massive PE.

Günay et al. (17) found that MPV levels were correlated with the pulmonary arterial occlusion level assessed by Computed tomography pulmonary arterial obstruction index ratio (CTPAOIR). These findings suggest that pulmonary arterial occlusion level that was the indicator of the massive PE can be predicted with the increased levels of MPV. Similarly, in our study, MPV levels were determined to be significantly higher in massive PE patients compared to patients with sub-massive or non-massive PE.

Vagdatli et al. (18) showed that MPV was increased during platelet activation. In that study, MPV levels were correlated with troponin. Similarly we also have determined a significant positive correlation between elevated troponin-I levels and increased MPV levels. However, we did not find any correlation between MPV levels and RV dysfunction.

In hemodynamically stable patients, RV dysfunction as observed by echocardiography has been shown to be able to identify patients with poor outcomes who might require more aggressive treatment. The availability of biomarkers like Troponin-I, BNP or NT-pro BNP which are able to identify patients with RV dysfunction early and to contribute these biomarkers to risk stratification is potentially important, especially when echocardiography assessment is not available $(19,20)$. Similarly we also have determined a significant positive correlation between elevated troponin-I levels and increased MPV levels. Regarding this correlation, among patients with higher MPV levels, clinicians should be alert about the worse prognosis.

In this study, higher MPV levels have been determined in massive and sub-massive PE patients compared with the non-massive PE patients. These results also support our hypothesis that MPV can predict disease severity in acute PE patients.

The limitations of the study have to be mentioned. First, this is a cross-sectional study and the potential causal relationship between PE and high MPV levels cannot be concluded. Second, we did not investigate the effect of anticoagulant treatment on MPV levels. Third, we did not follow the patients prospectively and did not compare the survivors and nonsurvivors MPV levels. Fifth, the 
distribution of age was different in our PE and control cases. However, this study was designed to show the association of MPV levels and severity of acute PE.

In conclusion; MPV as a potential marker of platelet activation may correlate with the extent of PE. However it seems not to be a significant predictor of mortality in acute PE. It is supposed that, in risk stratification of patients with acute PE, inserting MPV to the left ventricle dysfunction and increased bio-indicator combination may be helpful in deciding about the thrombolytic treatment. To better clarify that issue, further prospective studies with larger numbers are warranted.

\section{References}

1. Goldhaber SZ. Pulmonary embolism. Lancet 2004; 363: 1295-305.

2. Palmieri V, Gallotta G, Rendina D, et al. Troponin I and right ventricular dysfunction for risk assessment in patients with nonmassive pulmonary embolism in the Emergency Department in combination with clinically based risk score. Intern Emerg Med 2008; 3: 131-138.

3. Becattini C, Agnelli G. Acute pulmonary embolism: risk stratification in the emergency department. Intern Emerg Med 2007; 2: 119-129.

4. Masotti L, Righini M, Vuilleumier N, et al. Prognostic stratification of acute pulmonary embolism: focus on clinical aspects, imaging, and biomarkers. Vasc Health Risk Manag 2009; 5: $567-$ 575.

5. Gasparyan AY, Ayvazyan L, Mikhailidis DP, Kitas GD. Mean platelet volume: a link between thrombosis and inflammation? Curr Pharm Des 2011; 17: 47-58.

6. Rifaioglu EN, Sen BB, Ekiz O. Mean platelet volume and eosinophilia relationship in patients with bullous pemphigoid. Platelets. 2014; 25: 264267.

7. Hekimsoy Z, Payzin B, Ornek T, Kandoğan G. Mean platelet volume in Type 2 diabetic patients. J Diabetes Complications 2004; 18: 173-176.

8. Arslan E, Yakar T, Yavaşoğlu I. The effect of smoking on mean platelet volume and lipid profile in young male subjects. Anadolu Kardiyol Derg 2008; 8: 422-425.

9. Kostrubiec M, Labyk A, Pedowska-Włoszek J, et al. Mean platelet volume predicts early death in acute pulmonary embolism. Heart 2010; 96: 460465.

10. Torbicki A, Perrier A, Konstantinides S, et al. ESC Committee for Practice Guidelines (CPG). Guidelines on the diagnosis and management of acute pulmonary embolism: the Task Force for the Diagnosis and Management of Acute Pulmonary Embolism of the European Society of Cardiology (ESC). Eur Heart J 2008; 29: 2276-2315.

11. Mirzaie AZ, Abolhasani M, Ahmadinejad B, Panahi M. Platelet count and MPV, routinely measured but ignored parameters used in conjunction with the diagnosis of acute coronary syndrome: single study center in Iranian population, 2010. Med J Islam Repub Iran 2012; 26: 17-21.

12. Avramakis G, Papadimitraki E, Papakonstandinou D, et al. Platelets and white blood cell subpopulations among patients with myocardial infarction and unstable angina. Platelets 2007; 18: 16-23.

13. Tsiara S, Elisaf M, Jagroop IA, Mikhailidis DP. Platelets as predictors of vascular risk: is there a practical index of platelet activity? Clin Appl Thromb Hemost 2003; 9: 177-190.

14. Chung T, Connor D, Joseph J, et al. Platelet activation in acute pulmonary embolism. J Thromb Haemost 2007; 5: 918-924.

15. Ermiş H, Yucel N, Gulbas G, Turkkan S, Aytemur ZA. Does the mean platelet volume have any importance in patients with acute pulmonary embolism? Wien Klin Wochenschr 2013; 125: 381 385.

16. Varol E, Icli A, Uysal BA, Ozaydin M. Platelet indices in patients with acute pulmonary embolism. Scand J Clin Lab Invest 2011; 71: 163-167.

17. Günay E, Sarinc Ulasli S, Kacar E, et al. Can platelet indices predict obstruction level of pulmonary vascular bed in patients with acute pulmonary embolism? Clin Respir J 2014; 8: 33-40.

18. Vagdatli E, Gounari E, Lazaridou E, et al. Platelet distribution width: a simple, practical and specific marker of activation of coagulation. Hippokratia 2010; 14: 28-32.

19. Coutance G, Le Page O, Lo T, Hamon M. Prognostic value of brain natriuretic peptide in acute pulmonary embolism. Crit Care 2008; 12: R109.

20. Binder L, Pieske B, Olschewski M, et al. Nterminal pro-brain natriuretic peptide or troponin testing followed by echocardiography for risk stratification of acute pulmonary embolism. Circulation 2005; 112: 1573-1579. 